\title{
Immunological Tolerance to Pig-serum Partially Inhibits the Formation of Septal Fibrosis of the Liver in Capillaria hepatica infected Rats
}

\author{
Rodrigo Guimarães Andrade, Bruna Magalhães Gotardo, Bárbara Cristina A Assis, \\ José Mengel, Zilton A Andrade ${ }^{+}$
}

Laboratorio de Patologia Experimental, Centro de Pesquisas Gonçalo Moniz-Fiocruz, Rua Valdemar Falcão 121, 41295-001 Salvador, BA, Brasil

Systhematized septal fibrosis of the liver can be induced in rats either by repeated intraperitoneal injections of pig-serum or by Capillaria hepatica infection. The relationship between these two etiological factors, as far as hepatic fibrosis is concerned, is not known, and present investigation attempts to investigate it.

C. hepatica-induced septal fibrosis of the liver was considerably inhibited in rats previously rendered tolerant to pig-serum. Pig-serum-tolerant rats developed antibodies against pig-serum when infected with $\mathrm{C}$. hepatica, but this did not happen when the infection occurred in normal rats. On the other hand, anti-C. hepatica antibodies failed to recognize any epitope in pig-serum, by Western blot. However, no evidence of an immunological cross reactivity was found, at least at the humoral level. Alternatively, cell-mediated mechanisms may be involved, and further investigations are warranted.

Key words: pig-serum model - Capillaria hepatica - hepatic septal fibrosis

Rats infected with the helminth Capillaria hepatica invariably develop a peculiar type of hepatic fibrosis, 3040 days after inoculation. By the time the worms are usually dying and disintegrating, and while the focal necroinflammatory lesions around them are exhibiting evidences of resorption, septal fibrosis starts throughout the liver (Ferreira \& Andrade 1993). Morphologically, it has close similarity to the fibrosis experimentally induced in rats by repeatedly intraperitoneal injections of pig-serum or by its albumin fraction (Paronetto \& Popper 1966, Ballardini et al. 1985, Andrade 1991, Bhunchet et al. 1996). In both cases the histological picture is dominated by fine and long fibrous septa, that crisscross the hepatic parenchyma, forming a mosaic pattern, sometimes reminiscent of the pig liver, that progressively may evolve toward a final morphological picture of cirrhosis (Paronetto \& Popper 1966, Ferreira \& Andrade 1993). Fibrosis first connects portal spaces to portal spaces and runs within and along the peri-sinusoidal zone III area of the liver acinus, but is preceded neither by hepato-cellular necrosis, nor by overt chronic inflammation (Rubin et al. 1969). Such characteristics strongly suggest the existence of a stimulus to nonparenchymal hepatic cells, probably through an immunological mechanism (Nakano 1980, Tsukamoto et al. 1990, Santos et al. 2001).

Bhunchet et al. (1996) demonstrated that rats turned tolerant to pig-serum failed to develop septal fibrosis or antibodies against it, when repeatedly injected during adult

Financial support: Papes III (Fiocruz)

Corresponding author. Fax: +55-71-3652155. E-mail: zilton@cpqgm.fiocruz.br

Received 16 April 2004

Accepted 3 September 2004 life. As a control to their experiments, they showed that the pig-serum tolerant animals still maintained their capacity to respond with hepatic fibrosis when subjected to treatment with carbon-tetrachloride. However, it would be more interesting in such case, to use another model of septal fibrosis, with similar characteristics as those of the pig-serum model, but dependent upon a different etiology. Since specificity is a hallmark of immunological reactions, such attempt would be of crucial importance. On this regard, it is herein indicated the C. hepatica-model as an adequate candidate. In as much as the C. hepaticainduced fibrosis can be partially suppressed by a neonatal toleration method similar to that used for the pigserum model (Lemos et al. 2003).

The present investigation checks on the similarities between the pig-serum and C. hepatica models of septal hepatic fibrosis, by analyzing the behavior of the parasite infection in pig-serum tolerant rats. This would test whether there is specificity for the pig-serum model of hepatic fibrosis and would eventually contribute to the concept that some types of hepatic fibrosis indeed have an immunological basis.

\section{MATERIALS AND METHODS}

Experimental groups

Group I - Thirteen neonatal Wistar rats of both sexes, received intraperitoneal injections of whole pig-serum, twice a week, from the first day of life up to adult life. They received a total of 36 injections, starting with the dose of $0.05 \mathrm{ml}$, which was gradually increased up to $1 \mathrm{ml}$ by the 10th day of injection onward. Immediately in sequence, these 18-week old animals were submitted to a liver biopsy, followed by the administration, in five of the animals, of 300 embryonated eggs of $C$. hepatica, suspended in saline, and placed directly into the stomach by a gastric tube. The remaining eight animals continued to be injected 
with pig-serum up to the end of experiment. Forty days after infection, a new hepatic biopsy was performed in all animals. They were sacrificed 30 days later, 70 days after C. hepatica infection. Details about the obtaining of eggs and their counting appear elsewhere (Ferreira \& Andrade 1993).

Group II - a) control for fibrosis induced by pig-serum - Ten 8-week old intact Wistar rats, males and females, weighting approximately $200 \mathrm{~g}$, received intraperitoneal injections of $1 \mathrm{ml}$ of whole pig-serum, twice a week, totaling 26 injections. After that period they were submitted to a surgical liver biopsy. At the same time blood was taken for serological tests; $b$ ) control of $C$. hepatica infection - Four 8-week old Wistar rats, weighing $200 \mathrm{~g}$, males and females, were infected with approximately 300 embryonated eggs of $C$. hepatica, administered by gavage, and were sacrificed 40 days later.

All the animals were maintained in good housing conditions, with controlled temperature and humidity, in separated boxes according to sexes, with free access to a commercial balanced diet and water. The size of the inoculum was arbitrarily chosen within the range expected to regularly produce septal fibrosis (Oliveira \& Andrade 2001).

Pig-serum - The serum was obtained from the blood of one recently killed adult healthy pig. After blood coagulation at room temperature and centrifugation, the serum was collected, frozen in liquid nitrogen, and stored at $-70^{\circ} \mathrm{C}$ in several vials, each one being thawed at the moment of use. Only one batch was utilized throughout the experiments.

C. hepatica eggs - They were obtained from the livers of experimentally infected rats, around the 40th day after inoculation. The livers were washed in order to get rid of excess blood, homogeinized in an electrical blender, followed by several turns of washing with tap water and decantation, until the liquid above the sediment was completely clear. The clean immature eggs were kept moisturized with $0.5 \%$ formalin solution in a Petri dish, at room temperature $\left(26-28^{\circ} \mathrm{C}\right)$, for $28-30$ days, in order to embryonate. They were counted under the microscope and used in the dose of 300 eggs suspended in $0.5 \mathrm{ml}$ of distilled water per each animal.

Liver biopsy - With the animals under sodium pentobarbital general anesthesia, their abdomens were shaved and aseptically opened at the midline. The liver was exposed and a fragment of approximately 0.25 to $0.30 \mathrm{~g}$ was tied and removed. Recovery of the operated animals was uneventful.

Tissue preparation - Fragments obtained from biopsies and at the time of sacrifice of the animals were immediately fixed in neutral $10 \%$ formalin and routinely processed for paraffin embedding and cutting. Sections were stained with hematoxylin and eosin, picro-sirius-red method for collagen, PAS method, with and without previous diastase treatment, Gomori's silver impregnation for reticulin and the Perl's method for iron.

ELISA - This method was used to evaluate serum antibody levels either against pig-serum and $C$. hepatica. Sera were colleted from the tail vein at several occasions. For the animals injected since the first neonatal day with pig-serum, sera were obtained at the end of the toleration period and at the final period of $C$. hepatica infection. As for the control groups, sera were taken before and after either pig-serum treatment or $C$. hepatica infection. Detection of total Ig antibodies was made by using a goat anti-rat IgG conjugated to peroxidase (Sigma, St. Louis, MO, US). The plates were sensitized with $10 \mu \mathrm{g} / \mathrm{ml} / \mathrm{per}$ well of either $C$. hepatica-egg antigen or pig-serum, diluted in carbonate buffer, $\mathrm{pH}$ 9.9. Lecture was made on a microplate reader "Molecular devices-Thermomax" spectrophotometer (Sunyvalle, CA, US) under wave-length 450 $\mathrm{mm}$, connected to a computer with MDS-Soft Max with MDS-Soft Max. All sera were used at 1:1000 dilution in ELISA assays.

Western blot - C. hepatica antigens were prepared either from adult worms or from immature eggs. These materials were separately collected from the livers of experimentally infected mice. They were washed several times in distilled water, concentrated by centrifugation, and then homogenized, and suspended in saline. The samples utilized included these antigens, pig-serum, normal rat serum and sera from $C$. hepatica-infected rats. The samples were diluted in buffer ( $2 \%$ sodium dodecyl sulfate - SDS, $10 \%$ glycerol, $50 \mathrm{mM}$ Tris pH 6.8) plus $100 \mathrm{mM}$ mercaptoethanol, $0.1 \%$ bromophenol blue, and submitted to electrophoresis in 15\% polyacrylamide gel and 5\% stacking gel (Amresco Inc., Solon, OH, US). The gel was transferred to a nitrocellulose membrane for $2 \mathrm{~h}$ at $100 \mathrm{~V}$ (Novex - Novel Experimental Technology, San Diego, CA, US). The membranes were blocked with phosphate-buffered saline containing $0.3 \%$ tween and 5\% skimmed milk during $1 \mathrm{~h}$, under constant agitation. The membrane was then incubated with 1:650 C. hepatica-infected rat serum in blocking buffer, during $1 \mathrm{~h}$ under agitation. This was followed by incubation with rabbit peroxidase-conjugated anti-rat IgG (Sigma), diluted 1:650. Staining was accomplished by means of a peroxidase substrate-chromogen kit (Vector Laboratories, Inc., Burlingame, CA). The reaction was stopped with water.

Statistical analysis - The Kruskal-Wallis and the Dunn multiple comparison nonparametrical tests were used to compare serological data obtained from different experimental groups, considering $\mathrm{P}<0.05$ as significant.

\section{RESULTS}

All pig-serum tolerant animals developed infection when inoculated with embryonated $C$. hepatica eggs. The liver sections exhibited both disintegrating and well-preserved worms and eggs, within encapsulated focal lesions, which were sparsely distributed throughout the hepatic parenchyma. When compared with infected controls, these parasitic lesions were less numerous (Table), with diminished inflammatory cellular infiltration and peri-focal fibrosis. These lesions also contained several wellpreserved worms, which were exceptionally seen in nontolerant rats by the 40th day of infection. However, a clearcut difference was noted in the degree and distribution of septal fibrosis. The livers of $C$. hepatica-infected pigserum tolerant rats presented septal fibrosis in some focal areas of the liver only. Septa were thin and long, segregating areas of parenchyma of different sizes and appearance. Most of the time septa fibrosis was limited to a few 
TABLE

Semi quantitative evaluation of hepatic fibrosis and inflammation in rats treated with pig serum and/or infected with Capillaria hepatica

\begin{tabular}{|c|c|c|c|c|c|c|}
\hline \multirow[t]{2}{*}{ Groups } & \multirow[t]{2}{*}{ Description } & \multirow[t]{2}{*}{$\mathrm{N}$} & \multicolumn{3}{|c|}{ Fibrosis } & \multirow[t]{2}{*}{ Inflammation } \\
\hline & & & Present & Absent & Grade & \\
\hline I & Pig-serum tolerant $C$. hepatica-infected & 5 & 5 & 0 & $+/++$ & + \\
\hline I & Non-infected pig-serum tolerant & 8 & 0 & 8 & 0 & 0 \\
\hline II a & Pig-serum treated & 9 & 4 & 5 & ++ & 0 \\
\hline II $b$ & C. hepatica-infected & 4 & 4 & 0 & +++ & $++/+++$ \\
\hline
\end{tabular}

+ mild; ++ moderate; +++ marked

spurs radiating from the capsule of the focal parasitic lesions, while the rest of the liver parenchyma maintained its normal appearance (Fig. 1A). On the other hand, the livers from infected control rats exhibited septal fibrosis throughout the parenchyma, being more accentuated around focal parasitic lesions. Frequently, a morphologic
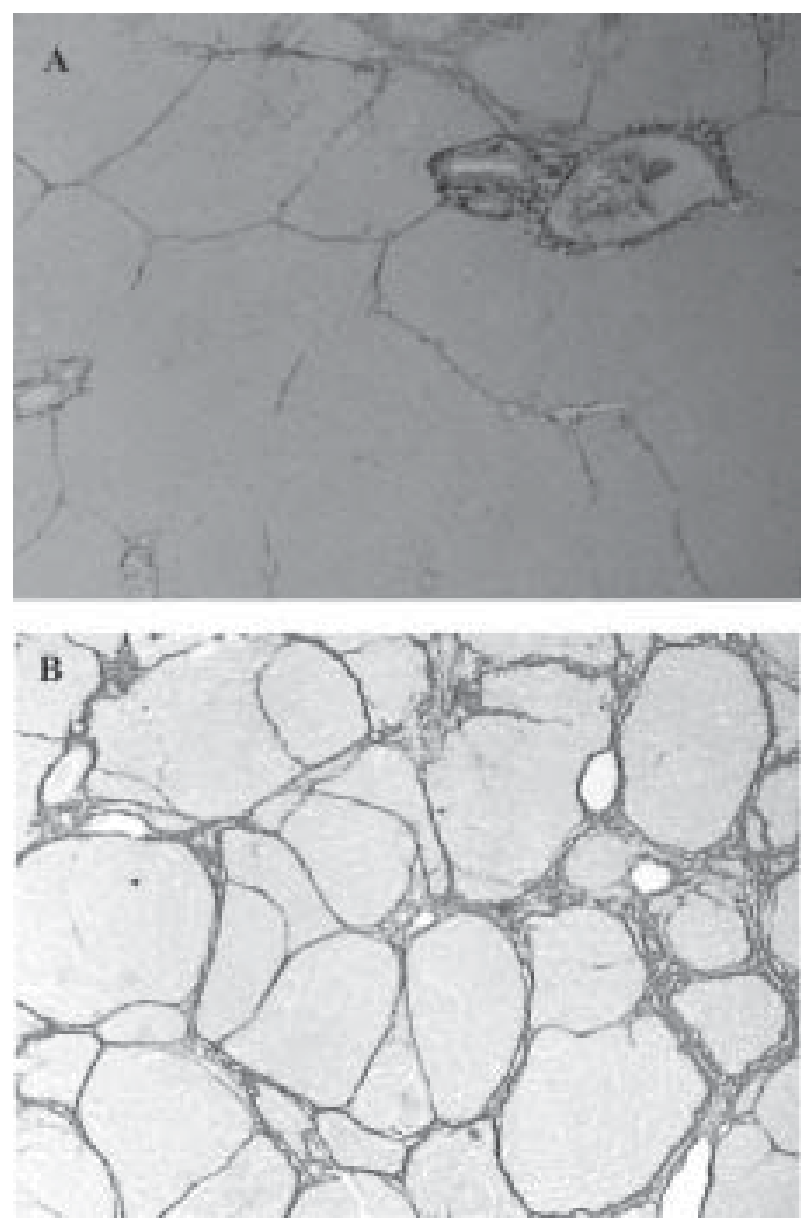

Fig. 1: septal fibrosis of the liver in a rat infected with Capillaria hepatica, as seen 40 days after infection. A: pig-serum tolerant rat. Septa are few, thin, and discontinued; B: non-tolerant rat. Septa are numerous and encircle irregular and nodular portions of the hepatic parenchyma, simulating a picture of cirrhosis. Picro-sirius-red staining method for collagen. X100 picture of cirrhosis was formed, with round hepato-cellular nodules delimited by fibrous septa (Fig. 1B).

Animals subjected to immunological tolerance with pig-serum did not exhibit serum antibodies, neither against pig-serum or $C$. hepatica antigens (Figs 2, 3). However, 40 days after being infected with $C$. hepatica, the pigserum tolerant animals presented serum antibodies against both pig-serum and $C$. hepatica. (Fig. 2). On the other hand, both antibodies were detected in the sera of normal adult rats after they were repeatedly treated with pig-serum (Figs 2, 3). However, the presence of antibodies against pig-serum was not detected in the sera of rats infected with $C$. hepatica only, as measured 40 days after inoculation (Fig. 2).

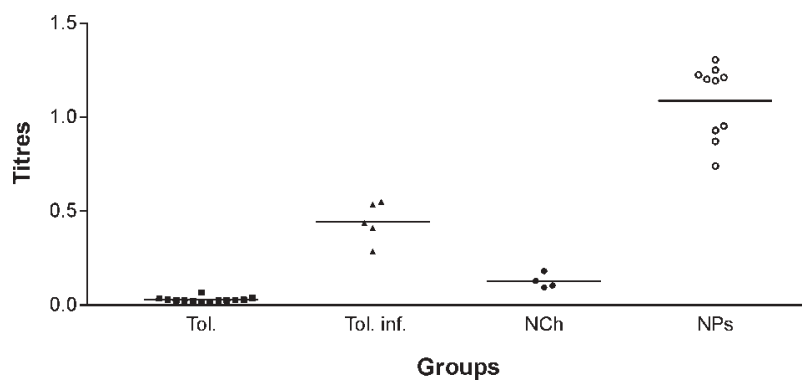

Fig. 2: total Ig against pig-serum (ELISA) in pig-serum tolerant rats: before (Tol.) and after Capillaria hepatica infection (Tol. inf.); and in normal rats: after $C$. hepatica infection $(\mathrm{NCh})$ and following repeated pig-serum administration (NPs) P > 0.05 Tol. vs Tol. inf.; P $>0.05$ Tol. vs NCh; P $<0.001$ Tol. vs NPs

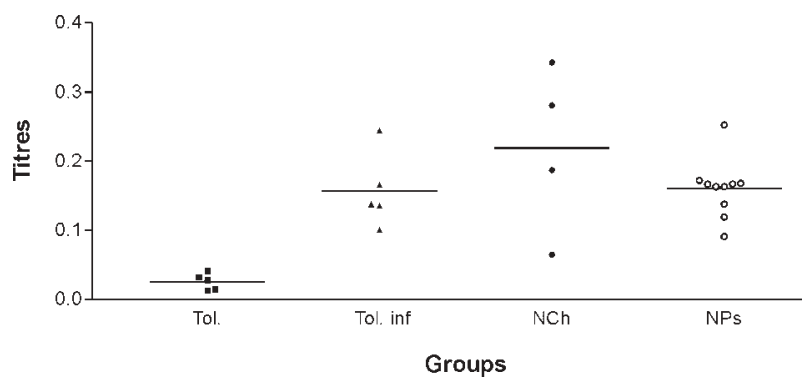

Fig. 3: total Ig against Capillaria hepatica (ELISA) in the sera of pig-serum tolerant rats (Tol.: tolerant non-infected), tolerant postC. hepatica infection (Tol. inf.), in normal non-tolerant rats (NCh: non-tolerant $C$. hepatica-infected rats) and in normal rats repeatedly injected with pig-serum (NPs) P > 0.05 Tol. vs Tol. inf.; P < 0.01 Tol. vs NCh; $\mathrm{P}<0.05$ Tol. vs NPs 
The antibodies present in the sera of $C$. hepaticainfected rats (Group II b) failed to recognize any antigenic fraction from the pig-serum by Western-blot.

As observed by histopathology, not a single animal receiving pig-serum injections from the first neonatal day until adult life, developed septal fibrosis of the liver. However, a moderate to severe degree of septal fibrosis did develop in four out of nine normal rats treated with pigserum since adult life. This group was reduced to nine animals because one of them died spontaneously near the end of the experiment and was not autopsied. Antibodies were present in the serum of all these treated animals, but no correlation was found between their levels and the degree of septal fibrosis, even when animals with and without fibrosis were compared.

All animals inoculated with embryonated $C$. hepatica eggs developed infection.

\section{DISCUSSION}

The present investigation confirmed and extended previously described observations concerned with the induction of liver fibrosis in adult rats receiving multiple injections of pig-serum (Paronetto \& Popper 1966, Ballardini et al. 1985, Bhunchet et al. 1996), and its absence when pig-serum administration started in the neonatal period (Bhunchet et al. 1996). In addition to maintaining their capacity to respond with hepatic fibrosis to carbon tetrachloride administration, as demonstrated by Bhunchet et al. (1996), pig-serum tolerant rats were still able to develop septal hepatic fibrosis, when subjected to a different etiologic agent, although to a reduced degree. In addition, it was also shown that long-term pig-serum administration, starting in the neonatal period, could profoundly influence the development of liver fibrosis, which usually accompanies the infection of rats with $C$. hepatica. The animals presented a less intense inflammatory infiltrate in response to the parasitic infection and an inhibition in the development of septal fibrosis of the liver. However, $C$. hepatica infection also raised up the levels of antibodies to pig-serum components in previously unresponsive rats. Higgins and Weiner (1988) have called attention to the fact that disease manifestations can be partially or completely suppressed in tolerant host in a dose-dependent manner, indicating that the state of tolerance is not a static one.

The upsurge of anti-pig-serum antibodies in previously tolerant rats infected with $C$. hepatica suggests that this helminth may share common antigenic epitopes with pig-serum. This primary hypothesis was ruled out by the demonstration that $C$. hepatica-infected rats do not produce specific antibodies to pig-serum components, although adult rats injected multiple times with pig-serum showed an antibody response to $C$. hepatica antigens. Also, in both experimental approaches rats produced specific antibodies to immunizing antigens. This set of experimental data strongly suggests that these different sets of antigens ( $C$. hepatica and pig-serum) do not share cross-reactive epitopes at the level of antibody recognition, as far as IgG antibodies are concerned. However, one may not rule out the possibility that such set of antigens may share T cell epitopes. Reactivity to immunoglo- bulin isotypes other that $\operatorname{IgG}$ should also be considered. Therefore, breaking of tolerance to pig-serum antigens by $C$. hepatica infection, as suggested in other experimental systems (Costalonga et al. 2002, Roep et al. 2002), may still be the explanation for this finding. Yet, the raise in antibody levels to pig-serum antigens in previously unresponsive mice, induced by infection with $C$. hepatica in the absence of further specific antigen administration is intriguing, and suggests that the rats have been, in fact, silenced primed for an immune response. In addition, the set of data showed in this study argues against a major role for antibodies in the genesis of liver fibrosis in this model, since animals presenting similar levels of antibodies, either to pig-serum components or $C$. hepatica, presented distinct histological aspects in relation to liver fibrosis and inflammatory response to the parasitic agent. Taken together, these provoking findings may stimulate the formulation of an alternative hypothesis to be considered. First of all, one should point out that the immune response to pig-serum antigens has a conventional aspect, which is the production of specific antibodies to pig-serum components, which apparently has no major impact in the modulation of liver fibrogenesis and a very peculiar, local or regional liver immune response that may lead to major changes in liver fibrogenesis without affecting the hepatocytes. The liver harbors conventional $\mathrm{T}$ cells, NK cells, gamma-delta T cells, and a special T cell lineage known as NK T cells (Kmiec 2001). It was shown that NK T cells secrete a number of different cytokines upon stimulation, including high amounts of IFN- $\gamma$ and IL-4 (Burdin et al. 1999, Takeda et al. 2000). More importantly, the pattern of NK T cell cytokine production may be modulated by chronic antigen stimulation (Yoshimoto $\&$ Paul 1994). In addition, liver NK T cells have been related to liver injury in a mouse model of autoimmune hepatitis (Chen \& Paul 1997) and in one study, NK T cells were involved with liver fibrosis in a mouse model of infection (Carvalho et al. 2002). Therefore, experiments aiming at characterizing the role of $\mathrm{NK} \mathrm{T}$ cells and conventional $\mathrm{T}$ cells are urgently required to better define their role in liver fibrogenesis and, in particular, in this experimental model.

\section{REFERENCES}

Andrade ZA 1991. Contribution to the study of septal fibrosis of the liver. Internat J Exper Pathol 72: 553-562.

Ballardini G, Faccani A, Beti S, Vasi V, Castaldini C, Biagini G, Garbisa S, Bianchi FB 1985. Sequential behaviour of intracellular matrix glycoproteins in an experimental model of hepatic fibrosis. Virchows Archiv (Cell Pathol) 49: 317324.

Bhunchet E, Eishi Y, Wake K 1996. Contribution of immune response to the hepatic fibrosis induced by porcine serum. Hepatology 23: 811-817.

Burdin N, Brossay L, Koezuka Y, Smiley ST, Grusby MJ, Gui M, Taniguchi M, Hayakawa K, Kronenberg M 1998. Selective ability of mouse CD1 to present glycolipids: alphagalactosylceramide specifically stimulates V alpha 14+ NK T lymphocytes. J Immunol 161: 3271-3281.

Burdin N, Brossay L, Kronenberg M 1999. Immunization with alpha-galactosylceramide polarizes CD1-reactive NK T cells towards Th2 cytokine synthesis. Eur J Immunol 29: 201420125. 
Carvalho CR, Lenzi HL, Correa-Oliveira R, Vaz NM 2002. Indirect effects of oral tolerance to ovalbumin interfere with the immune responses triggered by Schistosoma mansoni eggs. Braz J Med Biol Res 35: 1195-1199.

Chen H, Paul WE 1997. Cultured NK1.1+ CD4+ T cells produce large amounts of IL-4 and IFN-gamma upon activation by anti-CD3 or CD1. J Immunol 159: 2240-2249

Costalonga M, Hodges JS, Herzberg MC 2002. Streptococcus sanguis modulates type II collagen-induced arthritis in DBA/ 1J mice. J Immunol 169: 2189-2195.

Ferreira LA, Andrade ZA 1993. Capillaria hepatica: a cause of septal fibrosis of the liver. Mem Inst Oswaldo Cruz 88: 441-447.

Higgins PJ, Weiner HL 1988. Suppression of experimental autoimmune encephalomyelitis by oral administration of myelin basic protein and its fragments. J Immunol 140: 440-445.

Kmiec Z 2001. Cooperation of liver cells in health and disease. Adv Anat Embryol \& Cell Biol 161: 1-151

Lemos QT, Magalhães-Fernandes I, Andrade ZA 2003. Immunological basis of septal fibrosis of the liver in Capillaria hepatica-infected rats. Braz J Med Biol Res 36: 1201-1207.

Nakano M 1980. Early morphological changes of porcine serum-induced hepatic fibrosis. Acta Pathol Jpn 3: 415-422.

Oliveira RF, Andrade ZA 2001. Worm load and septal fibrosis of the liver in Capillaria hepatica-infected rats. Mem Inst Oswaldo Cruz 96: 1001-1003.

Paronetto F, Popper H 1996. Chronic liver injury induced by immunologic reactions. Cirrhosis following immunization with heterologous sera. Am J Pathol 40: 1087-1101.

Roep BO, Hiemstra HS, Schloot NC, De Vries RR, Chaudhuri A, Behan PO, Drijfhout JW 2002. Molecular mimicry in type 1 diabetes: immune cross-reactivity between islet autoantigen and human cytomegalovirus but not Coxsackie virus. Ann New York Acad Sci 958: 163-165.

Rubin E, Hutterer F, Popper H 1968. Experimental hepatic fibrosis without hepatocellular regeneration. A kinetic study. Am J Pathol 52: 111-119.

Santos AB, Tolentino Jr M, Andrade ZA 2001. Pathogenesis of hepatic septal fibrosis associated with Capillaria hepatica infection of rats. Rev Soc Bras Med Trop 34: 503-506.

Takeda K, Hayakawa Y, Van Kaer L, Matsuda H, Yagita H, Okumura K 2000. Critical contribution of liver natural killer T cells to a murine model of hepatitis. Proc Nat Acad Sci USA 97: 5498-5503.

Tsukamoto H, Matsuoka M, French SW 1990. Experimental models of hepatic fibrosis. A review. Sem Liver Dis 10: 5665.

Yoshimoto T, Paul WE 1994. CD4pos, NK1.1 pos T cells promptly produce interleukin 4 in response to in vivo challenge with anti-CD3. J Exper Med 179: 1285-1295. 
